Tanja Salem

\title{
Das Bildungsnetzwerk Ein Quadratkilometer Bildung Berlin-Neukölln: Ergebnisse der Abschlussevaluation
}

\section{Zusammenfassung}

2006 wurde in Berlin-Neukölln, im sogenannten Reuterkiez, der erste Standort des von der Freudenberg Stiftung für Schulen in schwieriger Lage entwickelten Programms Ein Quadratkilometer Bildung eröffnet und für zehn Jahre konzeptionelle und finanzielle Unterstützung durch verschiedene zivilgesellschaftliche und politische Partner für den Auf- und Ausbau eines Bildungsnetzwerks zugesagt. Das "Herzstück“ des Programms stellt die Pädagogische Werkstatt dar, ein eigenständiges, stadteilbezogenes und allen zugängliches Unterstützungssystem. Wie sich Ein Quadratkilometer Bildung BerlinNeukölln über die zehn Jahre entwickelt hat, war Gegenstand einer Abschlussevaluation; ausgewählte Ergebnisse, insbesondere zur Pädagogischen Werkstatt, werden im folgenden Beitrag vorgestellt.

Schlüsselwörter: Bildungsnetzwerke, Bildungslandschaften, Unterstützungssysteme, Pädagogische Werkstatt, Qualitätsentwicklung

\section{The Educational Network One Square Kilometre of Education Berlin-Neukölln: Results of the Final Evaluation}

\begin{abstract}
In 2006, One Square Kilometre of Education, a program for schools in deprived areas founded by the Freudenberg Foundation, started in Berlin-Neukölln. For ten years, conceptual and financial support from various civil society and political partners were pledged for the development of an educational network. The centrepiece of the program is the Pedagogical Workshop, an independent support system for educational institutions in the district. How One Square Kilometre of Education Berlin-Neukölln developed over the ten years, was the subject of a final evaluation. Central findings of the evaluation are presented, especially with regard to the Pedagogical Workshop.
\end{abstract}

Keywords: educational networks, support system, Pedagogical Workshop, quality development 


\section{Einleitung}

Ob Kinder und Jugendliche im deutschen Bildungssystem erfolgreich sind, hängt in hohem Maße von ihrer sozialen Herkunft ab (vgl. Autorengruppe Bildungsberichterstattung, 2016). Eine - keineswegs neue - bildungspolitische wie -praktische Maßnahme ist vor diesem Hintergrund der Aufbau „kommunaler Bildungslandschaften“, in denen die „für Bildung zuständigen Akteure [...] auf der Basis verbindlicher Strukturen [zusammenarbeiten]: Familie, Kinder- und Jugendhilfe, Schule, Kultur, Sport, Wirtschaft etc.“ Ziel ist die Förderung der individuellen Potenziale, denn „kein Kind dürfe verloren gehen“ (Deutscher Städtetag, 2007, S. 2).

Bildungslandschaften sollen somit zur Chancengleichheit im Bildungssystem beitragen.

Seit den 1990er/2000er-Jahren sind international wie auch in Deutschland verschiedene Programme und Projekte realisiert worden, die den Aufbau solcher Bildungslandschaften bzw. Bildungsnetzwerke unterstützen, z. B. „Lernen vor Ort“, „Lernende Region“, „Ganztägig lernen“ oder auch das Programm Ein Quadratkilometer Bildung der Freudenberg Stiftung mit inzwischen zehn Bildungsnetzwerken in unterschiedlichen Regionen Deutschlands. ${ }^{1}$

Die Diskussionen über Bildungslandschaften bzw. Bildungsnetzwerke bilden den Hintergrund des Beitrags. Im Zentrum stehen Ergebnisse der Abschlussevaluation von Ein Quadratkilometer Bildung Berlin-Neukölln ${ }^{2}$ (im Folgenden auch abgekürzt: km2 Berlin-Neukölln). ${ }^{3}$ Zunächst werden der Diskussions- und Forschungsstand zu Bildungslandschaften bzw. Bildungsnetzwerken und Unterstützungssystemen präsentiert und das Programm Ein Quadratkilometer Bildung vorgestellt. Es folgt die Darstellung der Anlage der Untersuchung und der Ergebnisse. Der Beitrag schließt mit einem knappen Fazit.

1 Von den zehn teilnehmenden Orten ist für die beiden ersten (Berlin-Neukölln 2006-2016 und Berlin-Moabit 2008-2018) die zehnjährige Programmphase beendet; die Netzwerke werden aber weiter gefördert. Auch die Freudenberg Stiftung unterstützt beide Bildungsnetzwerke beratend und finanziell, und in Berlin-Neukölln beteiligt sich der Bezirk durch die Finanzierung der Räume für die Pädagogische Werkstatt. Zudem werden zusätzliche private und öffentliche Fördermittel akquiriert, ohne die viele Aktivitäten nicht möglich wären.

2 Verfügbar unter: http://www.ein-quadratkilometer-bildung.eu/chronik/vorstellung-eva-2017; Zugriff am 05.03.2018.

3 Mein herzlicher Dank gilt Sabrina Wangenheim, die insbesondere bei der Transkription von Interviewmaterial und bei der Aufbereitung von Forschungsliteratur unverzichtbare Unterstützung leistete. 


\section{Bildungsnetzwerke und Unterstützungssysteme - Anmerkungen zum Forschungsstand}

Bildungslandschaften (resp. Bildungsnetzwerke $)^{4}$ werden als „langfristige, professionell gestaltete, auf gemeinsames, planvolles Handeln abzielende, kommunalpolitisch gewollte Netzwerke zum Thema Bildung" (Bleckmann \& Durdel, 2009, S. 12) definiert. Raum in einem weit gefassten Sinne (Löw, 2010) und Bildung werden als eng verknüpft und in Wechselwirkung stehend betrachtet (Million, Coelen \& Heinrich, 2017). Für Kinder und Jugendliche sollen durch Bildungslandschaften Bildungs- und Lerngelegenheiten geschaffen werden, die ihren Bedarfen entsprechen (vgl. Bollweg \& Otto, 2011; BMFSFJ, 2006).

Aus ökosystemtheoretischer Perspektive ${ }^{5}$, die in vielen Studien zu Bildungsnetzwerken forschungsleitend ist, werden nicht nur die (professionellen) Voraussetzungen von Personen als bedeutsam für die Förderung der Entwicklung und Bildung junger Menschen angesehen, sondern auch die Gestaltung der Interaktion und Kooperation zwischen Bildungsinstitutionen (z. B. Kita und Schule) respektive Personen, d.h. pädagogischen Fachkräften und Eltern untereinander und mit dem Kind. Darüber hinaus sind die Merkmale der Bildungsinstitutionen, z. B. die räumliche und personelle Ausstattung, die Qualität der Bildungsangebote, die Gestalt des direkten Umfeldes, in dem junge Menschen aufwachsen, bildungsadministrative und gesamtgesellschaftliche Bedingungen einflussreich (vgl. Bronfenbrenner \& Morris, 2006; Rimm-Kaufman \& Pianta, 2000).

Damit Bildungsnetzwerke entstehen und Bildungseinrichtungen sich in diesen weiterentwickeln, bedarf es der Unterstützung, z. B. durch Fortbildungsinstitute oder Schulentwicklungsberatung. Angemerkt wird auch, dass das Konzept der Unterstützung im Rahmen von Bildungsnetzwerken weiter zu schärfen sei. Erste Ansätze dazu, so z.B. das regionale Netzwerkmanagement, sind schon gegeben. Untersuchungen zeigen, dass ein solches maßgeblich die Entwicklung sowie die Ergebnisse, die im Netzwerk erreicht werden, beeinflusst (vgl. Otto, Sendzik, Järvinen, Berkemeyer \& Bos, 2015). Ebenfalls liegen professions- und transfertheoretisch fundierte Modelle vor, die die Professionalisierung von Fachkräften durch Unterstützungssysteme in den Mittelpunkt stellen. Diese wiederum kann zu einer verbesserten Bildungspraxis und damit zum Bildungserfolg junger Menschen beitragen (vgl. Järvinen, Sendzik, Sartory \& Otto, 2015).

Den Untersuchungen zu den inhaltlichen Schwerpunktsetzungen in Bildungsnetzwerken zufolge werden vor allem Themen wie fachbezogene Unterrichtsentwicklung,

4 Bildungslandschaften und Bildungsnetzwerke sind nicht eindeutige Bezeichnungen und werden durchaus auch synonym verwendet; im Folgenden wird mit dem Begriff Bildungsnetzwerk gearbeitet.

5 Siehe zu weiteren theoretischen Zugängen im Überblick Berkemeyer \& Bos (2010). 
die Vernetzung von Bildungseinrichtungen, die anschlussfähige Gestaltung von Übergängen im Bildungssystem und die Individualisierung von Bildung bearbeitet (vgl. Jungermann, Manitius \& Berkemeyer, 2015).

Kritisch angemerkt wird, dass, auch wenn Bildungsnetzwerke ebenso wie entsprechende Unterstützungssysteme mittlerweile relativ verbreitet sind, der damit verbundene Anspruch, dass Kinder und Jugendliche durchgängig auf ihrem Bildungsweg unterstützt werden und eben „nicht verloren gehen“, noch nicht eingelöst sei (Baumheier, Fortmann \& Warsewa, 2013). Hier setzt das Programm Ein Quadratkilometer Bildung an; es soll mit seinem Ansatz einen Beitrag zur Frage der durchgängigen Unterstützung von Kindern von der Kita bis in den Sekundarbereich leisten.

\section{Ein Quadratkilometer Bildung}

Das Programm Ein Quadratkilometer Bildung wurde von der Freudenberg Stiftung, der Karl-Konrad-und-Ria-Groeben-Stiftung und der RAA (Regionale Arbeitsstellen für Bildung, Integration und Demokratie e.V.) Berlin in Zusammenarbeit mit der Berliner Senatsverwaltung für Bildung, Wissenschaft und Forschung für Bildungsstandorte in sozial schwieriger Lage entwickelt. Das erste Bildungsnetzwerk startete 2006 im Reuterkiez Berlin-Neukölln. Bis 2015 wurden sukzessive neun weitere Bildungsnetzwerke in sozial benachteiligten Stadtteilen in sechs Bundesländern eingerichtet. Programmträger ist die Freudenberg Stiftung, die ihrerseits Kooperationsverträge mit dem jeweiligen lokalen Programmträger, dem Land und der Kommune und gegebenenfalls lokalen Stiftungen abgeschlossen hat. Das Programm ist in seinen Grundsätzen und seiner Grundstruktur überregional ausgerichtet, aber zugleich ist es so gefasst, dass es den jeweiligen Bedingungen vor Ort angepasst werden kann. Dabei wird stets darauf geachtet, schon bestehende Unterstützungsstrukturen einzubeziehen und den Aufbau von Parallelstrukturen zu vermeiden.

Ziel des Programms ist die Begleitung bzw. Unterstützung der Kinder und Jugendlichen über ihre gesamte Bildungsbiographie. Ein Quadratkilometer Bildung verfolgt hierzu einen kleinräumigen Ansatz, bei dem die Bildungswege der Kinder - räumlich wie institutionell - im Zentrum stehen. So wird das Bildungsnetzwerk an jedem Ort um die sogenannte Schlüsselgrundschule aufgebaut. Von ihr ausgehend werden nach und nach andere Bildungseinrichtungen einbezogen, z. B. Kindertageseinrichtungen (Kitas), vor allem solche, aus denen erfahrungsgemäß besonders viele Kinder in die Schlüsselgrundschule wechseln, Einrichtungen der Kinder- und Jugendarbeit und je nach den lokalen Bedingungen - auch Büchereien, Migrantenselbstorganisationen oder auch - wie im Fall von Ein Quadratkilometer Bildung Neubrandenburg - die im Kiez aktiven Tagesmütter. Entscheidend für die Beteiligung ist, dass die Institutionen 
für die Probleme vor Ort im Hinblick auf die Bildung von Kindern und Jugendlichen sensibel sind und aktiv an positiven Veränderungen arbeiten wollen.

Das „Herzstück“ des Bildungsnetzwerks ist die sogenannte Pädagogische Werkstatt. Ihr Auftrag ist es, institutionelle wie personelle Ressourcen im Quartier bzw. Stadtteil aufzuspüren und für die Qualitätsentwicklung in den Kitas, Schulen, Jugendeinrichtungen, aber auch für die Eltern und Lehrkräfte usw. nutzbar zu machen.

Die Pädagogische Werkstatt weist drei konzeptuelle Merkmale auf: (1) eigene Arbeitsräume mitten im Quartier, (2) Praxisbegleiter ${ }^{\star}$ innen, die als change agents fungieren und zu deren Aufgaben u.a. die Organisation und Moderation von Klausurveranstaltungen zum Thema Schulentwicklung und zu interinstitutionellen Kooperationsformaten sowie der Organisation von Fortbildungen für die pädagogischen Fachkräfte gehören, und (3) ein Entwicklungsbudget, d.h. finanzielle Mittel, die die Praxisbegleiter*innen frei verwalten können, um ihrem Auftrag nachzugehen. Träger der Pädagogischen Werkstatt ist jeweils eine gemeinnützige Agentur oder eine Bürgerstiftung. Ort, Personal und Entwicklungsbudget werden aus privaten, kommunalen und Landesmitteln finanziert (Wenzel, 2009).

\section{Ein Quadratkilometer Bildung in Berlin-Neukölln}

Die Entscheidung, im Berliner Reuterkiez das erste Bildungsnetzwerk zu etablieren, wurde auf der Basis der Schulleistungs- und Sozialdaten getroffen. Im Reuterkiez, ca. 70 Hektar groß, leben knapp 20.000 Personen. Trotz positiver sozialer Veränderungen seit Beginn des Programms 2006 steht der Kiez noch heute vor sozialen Herausforderungen: Kinderarmut, Verknappung von Wohnraum und steigende Mieten. Etwa ein Fünftel der Bewohner*innen bezieht Transferleistungen; bei den unter 15-Jährigen sind es 56,1 Prozent. Der Arbeitslosenanteil liegt mit 8,42 Prozent über dem Berliner Durchschnitt (6,4\%), und in den vier Schulen des Quartiers sind über 75 Prozent der Schüler*innen von Lernmittelzuzahlung befreit. ${ }^{6}$

Als Schlüsselgrundschule wurde 2006 die Franz-Schubert-Schule ausgewählt. Ausschlaggebend für die Wahl war, dass sie schon zuvor initiativ geworden war, um ihren Schüler ${ }^{\star}$ innen bessere Bildungschancen zu eröffnen. 2008 fusionierte sie mit zwei Schulen des Sekundarbereichs und konstituierte sich als Gemeinschaftsschule auf dem Campus Rütli $\mathrm{CR}^{2}{ }^{7}$ Zum Zeitpunkt der Evaluation gehörten alle Bildungs-

6 Verfügbar unter: http://www.reuter-quartier.de/Unser-Quartier.154.0.html; Zugriff am 05.03. 2018.

7 Näheres zum Campus Rütli $\mathrm{CR}^{2}$ findet sich unter: http://campusruetli.de/konzept/; Zugriff am 11.06.2018. Einen Einblick in die Geschichte und aktuelle Entwicklungen gibt URL: https://mediendienst-integration.de/artikel/campus-ruetli-schule-ein-quadratkilometer-bil dung-vielfalt-lernen.html; Zugriff am 11.06.2018. 
einrichtungen des Campus Rütli $\mathrm{CR}^{2}$, d.h. die Gemeinschaftsschule, eine Jugendeinrichtung, zwei Kitas und eine Elterninitiative, zum Bildungsnetzwerk sowie vier weitere Kitas, zwei weitere Einrichtungen der Kinder- und Jugendarbeit und zwei Kooperationsschulen. Außerdem wurde mit dem Quartiersmanagement zusammengearbeitet. Die Pädagogische Werkstatt wurde 2007 eröffnet, zunächst mit einem Praxisbegleiter; sukzessive wurden vier weitere Praxisbegleiterinnen eingestellt; sie bilden das multiprofessionelle Team.

Im Unterschied $\mathrm{zu}$ anderen Untersuchungen (vgl. Million et al., 2017; Duveneck, 2016) hat die Evaluation von km2 Berlin-Neukölln das Programm und die Pädagogische Werkstatt (Wenzel, 2009) als Unterstützungssystem für die Entwicklungen im Bildungsbereich im Berliner Reuterkiez in den Mittelpunkt gerückt.

\section{Ziel der Evaluation, methodisches Vorgehen und Datengrundlage}

Für die Evaluation am Ende der zehnjährigen Projektphase berief die Freudenberg Stiftung einen Arbeitskreis ein, der die Ziele und Fragestellungen der Evaluation bestimmte. Ihm gehörten Vertreter*innen der Stiftung, der Senatsverwaltung für Bildung, Jugend und Familie (Schulaufsicht Region Neukölln), der Senatsverwaltung für Stadtentwicklung und Wohnen sowie Mitglieder der Pädagogischen Werkstatt Berlin-Neukölln an. Ziel der Evaluation war es, die Entwicklungen in den zehn Jahren zu beschreiben, sie aus Sicht der Beteiligten, aber auch vor dem Hintergrund aktueller Forschung zu bewerten und die Elemente von Ein Quadratkilometer Bildung zu identifizieren, die dauerhaft in den Stadtteil und gegebenenfalls in andere Bildungsnetzwerke übertragen werden sollten. Dazu sollte geklärt werden, inwiefern eine durchgängige Begleitung der Bildungsprozesse von Kindern und Jugendlichen in km2 Berlin-Neukölln erreicht worden sei, welche Bedingungen als für diese Entwicklungen förderlich und welche als hinderlich anzusehen seien und welchen Beitrag die Pädagogische Werkstatt als Unterstützungssystem vor Ort geleistet habe.

Für die Durchführung der Evaluation wurden drei Datenquellen genutzt:

1. statistische Daten zur Bildungssituation von Kindern und Jugendlichen im Reuterkiez;

2. zentrale Dokumente des Projekts (z. B. Informationsbroschüren, Sitzungsprotokolle, Darstellungen des Programms, z. B. in PowerPoint-Präsentationen) und

3. qualitative leitfadengestützte Interviews mit Personen aus allen Institutionstypen, die aktiv an km2 Berlin-Neukölln mitgewirkt haben. 
Die Analyse der statistischen Daten, ${ }^{8}$ z. B. zu den Übergangszahlen von der Kita in die Grundschule(n), den Schulabbruchsquoten, zur Quote des Mittleren Schulabschlusses, sowie die Analyse von 35 zentralen Dokumenten erfolgten im Winter 2015, und die 31 leitfadengestützten Einzel- und Gruppen-Interviews mit 57 Schlüsselpersonen wurden 2016/17 geführt. Interviewt wurden Leitungskräfte, frühpädagogische Fachkräfte, Lehrkräfte, Mitarbeiter*innen von Stadtteilinitiativen bzw. -projekten, Eltern, eine Wissenschaftlerin und die Praxisbegleiter*innen. Der Leitfaden bezog sich auf folgende thematische Schwerpunkte: Beteiligung an $\mathrm{km} 2$ Berlin-Neukölln, institutionelle Entwicklungen, Vernetzung und interinstitutionelle Kooperation, Funktion der Pädagogischen Werkstatt und weiterer Unterstützungssysteme, Auswirkungen des Projekts auf die Beteiligten und den Sozialraum/ den Kiez, Gelingensbedingungen und Hürden, Zukunftsperspektiven, subjektive Deutungen der Ergebnisse der Analyse der schulstatistischen Daten. Die Auswertung erfolgte computergestützt entsprechend der inhaltlich strukturierenden qualitativen Inhaltsanalyse (Kuckartz, 2014). Das Kategoriensystem wurde deduktiv-induktiv entwickelt, orientiert an dem oben dargestellten Programmkonzept, der Forschungsliteratur und dem Interviewleitfaden.

Insgesamt wurden 14 Hauptkategorien gebildet (zusätzlich zu den Kategorien des Leitfadens: Rahmenbedingungen, Initiativen extern, Angebote km2 Berlin-Neukölln, Zusammenarbeit mit Eltern, Individualisierung) und im Laufe der Analyse durch Subkategorien ausdifferenziert. Wie bei der gewählten Methode üblich, wurden Beschreibungen der Hauptkategorien und Subkategorien verfasst und Ankerzitate definiert, um die Aussagen der Befragten den entsprechenden Kategorien zuordnen zu können.

Wie in Abschnitt 2 dargestellt, wird davon ausgegangen, dass Bildungsnetzwerke beim Aufbau und bei ihrer Entwicklungsarbeit Unterstützung benötigen; im Programm Ein Quadratkilometer Bildung wurde die Pädagogische Werkstatt als die Instanz konzeptualisiert, die dies leisten soll. Vor diesem Hintergrund wird im Folgenden die Funktion der Pädagogischen Werkstatt für das Bildungsnetzwerk im Bereich der Individualisierung von Bildungsprozessen als ein Ausschnitt aus den Evaluationsergebnissen (Salem, 2018) vorgestellt, und zwar am Beispiel der in $\mathrm{km} 2$ Berlin-Neukölln entwickelten Instrumente zur Beobachtung, Dokumentation und Reflexion der Lernprozesse von Kindern und Jugendlichen. ${ }^{9}$ Dabei wird der Blick auf die im Programm definierten Merkmale der Pädagogischen Werkstatt (Ort,

8 An dieser Stelle wird aufgrund des Fokus des Artikels nicht auf die Ergebnisse der statistischen Datenanalyse eingegangen. Sie werden an anderer Stelle publiziert. Verwiesen sei auch auf die Evaluationsberichte zur Gemeinschaftsschule. Verfügbar unter: https://www.berlin. de/sen/bildung/schule/bildungswege/gemeinschaftsschule/; Zugriff am 12.06.2018.

9 Es wurden weitere Veränderungen durch die Unterstützung der Pädagogischen Werkstatt erzielt, die an dieser Stelle nicht ausführlich dargestellt werden können. Sie sind auf der Programmwebsite nachzulesen. Verfügbar unter: http://ein-quadratkilometer-bildung.org/wo/ berlin-neuk\%C3\%B6lln; Zugriff am 16.03.2018. 
Praxisbegleiter*innen, Entwicklungsbudget), ihre Ausgestaltung, ihr Zusammenspiel mit dem und ihre Bedeutung für das Bildungsnetzwerk gerichtet. Basis des folgenden Abschnitts sind die Aussagen zu den Kategorien „Pädagogische Werkstatt“ und zu „Dokumentations- und Reflexionsinstrumente“ als Subkategorie von „Individualisierung“.

Unter der Kategorie „Pädagogische Werkstatt“ wurden in einem Analyseschritt (von mehreren) entlang des Programmkonzepts Aussagen zu Rahmenbedingungen (z. B. Träger), zum Raum/Ort, zu Merkmalen der Praxisbegleiter*innen, zu Handlungsansätzen der Praxisbegleiter ${ }^{\star}$ innen und zum Entwicklungsbudget eingeordnet, ${ }^{10}$ Aussagen zur Beschreibung, Entwicklung und Nutzung der Instrumente unter „Dokumentations- und Reflexionsinstrumente“. Mit der gewählten Analysemethode konnten Zusammenhänge zwischen diesen beiden Kategorien identifiziert werden, auf die im nächsten Abschnitt genauer eingegangen wird.

\section{Ergebnisse}

Im Verlauf der Arbeit vor Ort hat sich ergeben, dass in km2 Berlin-Neukölln schwerpunktmäßig Individualisierung bearbeitet worden ist. Das war im Programm nicht als solches vorgesehen, sondern es ergab sich im Arbeitsprozess und aus den nach und nach erkannten lokalen Problemen, Bedarfen und Potenzialen. Die Entwicklungen betrafen - im Kern ausgehend von der Schlüsselgrundschule z. B. das Schulprogramm, das mit der Schulfusion (s.o.) neu zu erarbeiten war, die Einführung des Jahrgangsübergreifenden Lernens (JüL) ${ }^{11}$ in der gesamten Grundstufe, die Erarbeitung und Implementierung von Instrumenten zur Beobachtung, Dokumentation und Reflexion der Lernprozesse von Kindern und Jugendlichen in der Grundstufe und im Sekundarbereich (das Lernentwicklungs-Portfolio „Meine Lernreise"12 und das Logbuch zur Unterstützung des selbstgesteuerten Lernens) sowie hieran anschlussfähige Instrumente für den Übergang Kita-Grundschule (sogenannte Einlegeblätter für das Berliner Sprachlerntagebuch) ${ }^{13}$ und für die Krippe (Eingewöhnungsbuch für die ersten Tage in der Krippe).

$10 \mathrm{Zu}$ dieser Kategorie wurden weitere Subkategorien gebildet. Die Ergebnisse werden im internen Evaluationsbericht und in weiteren Artikeln vorgestellt.

11 JüL ist in Berlin 2004 in der Schulanfangsphase verpflichtend eingeführt worden. Seit 2010 können die Schulen wieder selbst entscheiden, ob sie JüL praktizieren wollen.

12 In zwölf Rubriken wird die Entwicklung über die Grundstufe hinweg in den verschiedenen Lernbereichen der Schule dokumentiert und reflektiert, ebenso das Selbstbild des Kindes und dessen Lernprozess. Zum Zeitpunkt der Evaluation bestanden Bestrebungen, das Portfolio sowie JüL auch im Sekundarbereich einzuführen.

13 Das Berliner Sprachlerntagebuch ist das einzige Instrument im Elementarbereich, das jede Kita in Berlin verbindlich und unabhängig von ihrem Träger einsetzt und das mit Einverständnis der Eltern an die Schule übergeben wird. Dokumentiert werden (schulrelevante) sprachliche Fähigkeiten und mit den Einlegeblättern weitere (schulrelevante) Fähigkeiten. 
Um Individualisierung als leitendes Prinzip für die Unterrichts- und Schulentwicklung zu verankern, wurden eine enge Kooperation zwischen Schulleitung und Praxisbegleitung sowie deren Mitwirkung in den schulischen Gremien und die (Weiter-)Entwicklung und (Selbst-)Evaluierung des Schulprogramms durch pädagogische Fachkräfte und auch Eltern etabliert.

In den Interviews wurde von allen befragten Personen(gruppen) betont, wie wichtig die Arbeit der Pädagogischen Werkstatt für die Veränderungen gewesen sei. Insbesondere die Multiprofessionalität des Praxisbegleiter-Teams wurde positiv hervorgehoben - zum einen hinsichtlich der so gebündelten professionellen Kompetenz und zum anderen auch, weil jede Institution sich repräsentiert gefühlt habe.

Die Praxisbegleiter*innen identifizierten ihrerseits durch Beobachtung und (Selbst-) Evaluationsinstrumente problematische Bedingungen im Hinblick auf die Bildungsprozesse von Kindern und Jugendlichen wie auch Ressourcen von pädagogischen Fachkräften; sie griffen Ansätze zur Veränderung auf und entwickelten und erprobten sie zusammen mit den jeweils Verantwortlichen weiter. Sie initiierten und organisierten Austausch und Kooperation und moderierten und dokumentierten darüber hinaus Entwicklungsprozesse, insbesondere in der Schule. Ihre Interventionen bezogen sich auch auf verschiedene interinstitutionelle Kooperationsformate, z. B. auf die Arbeitsgruppen zur Erstellung von Instrumenten zur Dokumentation der Lernprozesse von Kindern. Sie brachten sich stets mit eigenen Ideen und Fachwissen ein, aber immer so, dass das Aufgreifen und Unterstützen vorhandener Vorstellungen und Ressourcen im Vordergrund stand und die pädagogischen Fachkräfte und Leitungskräfte die Veränderungen in ihren Einrichtungen selbst gestalten konnten.

So wurde z.B. 2008/2009 von den Praxisbegleiter*innen mittels des von ihnen entwickelten Instruments „Basisdatenerhebung“ (Meinz \& Wenzel, 2012) aufgedeckt, dass nur eine geringe Anzahl von Kindern aus den direkt umliegenden Kitas auf die Schlüsselschule wechselte.

Um diesbezüglich eine positive Veränderung zu erzielen, wurde unter anderem das schulische Portfolio als Baustein individuellen Lernens entwickelt. Es wird seit 2011 in der gesamten Grundstufe eingesetzt. Zurückzuführen ist die Entwicklung darauf, dass ein Praxisbegleiter bemerkt hatte, dass eine der Grundschullehrkräfte mit einem Portfolio arbeitete, aber niemand sonst; im Interview schilderte diese Lehrerin:

LK2: „Na, und hat halt/ ich habe halt IMMER schon diese Ideen gehabt, ne, Portfolio. Ich habe das auch in meiner Klasse gemacht, schon mit einem Portfolio gearbeitet. Und die haben halt einfach/ also in Person halt [PB1]: ,Ja, SUPERIDEE. Woran scheitert es? Was

Verfügbar unter: https://www.berlin.de/sen/jugend/familie-und-kinder/kindertagesbetreuung/fachinfo/\#sprache; Zugriff am 05.03.2018. 
| Tanja Salem

braucht ihr, damit ihr es umsetzen könnt?' Und da war endlich einer, der gefragt hat: Was brauchst du dafür?" (Lehrkräfte Jg. 4-6 \$78)

Die Initiative der Lehrerin wurde vom Praxisbegleiter aufgegriffen, und er initiierte und begleitete die Erarbeitung des Portfolios, an der mehrere Lehrkräfte mitwirkten. Über das Entwicklungsbudget konnten die grafische Gestaltung finanziert und der Druck sowie die Verteilung an die Schüler*innen gesichert werden. Seit 2011 wird das Portfolio in jeder Lerngruppe der Grundstufe eingesetzt, womit ein zentraler Anspruch von Individualisierung eingelöst wird, nämlich die Lernentwicklung des Kindes in den Mittelpunkt zu stellen.

Mit der Einführung des Portfolios veränderte sich zugleich die Zusammenarbeit mit den Erziehungsberechtigten der Kinder, denn zur Arbeit mit dem Portfolio gehört, dass Lehrkräfte Lernentwicklungsgespräche gemeinsam mit den Eltern und deren Kindern führen. Eine Mutter mit arabischsprachigem Hintergrund, die sich auch im Rahmen einer Variante von „Rucksack-Kita“ 14 als Elternbegleiterin in $\mathrm{km} 2 \mathrm{Berlin}$ Neukölln engagierte, bewertete die Gespräche wie folgt:

EB: „[...] Weil, wenn ich sehe, dass etwas fehlt, dass ich etwas machen muss für mein Kind. Das finde ich auch, ja, es ist SEHR gut, diese Entwicklung. Ja. [...]" (Elternbegleiterin $\$ 134$ )

In vielen Interviews wird auch betont, dass die Einführung des Portfolios sich nicht nur nach innen, sondern auch nach außen - in den "Sozialraum“ - positiv ausgewirkt habe; u.a. sei die Schule für bildungsorientierte Eltern attraktiver geworden, so die Schulleitung im Interview:

SL1: „Ja, aber es hatte eine wirklich große Signalwirkung ins Innere und ins Äußere, also auch in den Sozialraum hinein. Da ist eine Schule, die setzt den Punkt. Das war eigentlich der Beginn der vielen, dann deutschen Eltern, bildungsaffinen Eltern in der Grundstufe so [...]." (Schulleitung $1 \$ 121)$

Infolge der Arbeit mit dem Portfolio als einem wesentlichen Baustein der Schulentwicklung hat sich laut Schulleitung die Zusammensetzung der Schülerschaft

14 Rucksack-Kita ist ein mehrsprachiges Sprach- und Familienbildungsprogramm, das in Nordrhein-Westfalen entwickelt wurde. Zentrales Element ist, dass mehrsprachige sogenannte Elternbegleiter*innen andere mehrsprachige Eltern in den verschiedenen Fragen, die mit dem Aufwachsen und der Bildung der Kinder zusammenhängen, unterstützen. Gearbeitet wird in der Familiensprache und in Deutsch. In km2 Berlin-Neukölln wurde es in einer abgewandelten Form in einer Kita umgesetzt. Die Elternbegleiterin wurde durch die Pädagogische Werkstatt unterstützt. Verfügbar unter: http://www.kommunale-integrationszentren-nrw.de/rucksack-1; Zugriff am 05.03.2018. 
verändert, und die Anmeldezahlen in der Grundstufe haben sich im Vergleich zum Beginn von km2 Berlin-Neukölln wesentlich verbessert. ${ }^{15}$

Zudem wurde ausgehend von der Schule eine pädagogisch-methodische Verbindung mit km2 Bildung Kitas hergestellt, und zwar durch eine adaptierte Form des Portfolios, d.h. durch die Einfügung von Einlegeblättern für das Berliner Sprachlerntagebuch. Aus Sicht einer Kita-Leitung stellte sich der Beginn des Transferprozesses wie folgt dar:

KL2: „Also, jedenfalls haben auf irgendeiner Veranstaltung sie dann diese Einlagen oder dieses Portfolio uns vorgestellt und wir waren davon so begeistert, dass wir gesagt haben [...] , Na, Mensch, wenn wir eben auch so Blätter entwickeln und die dann zum Beispiel in unser Sprachlerntagebuch packen, dann können die Kinder, wenn sie dann in die Schule kommen, hier wieder etwas wiederentdecken.' Und dann hat sich eine Arbeitsgruppe gebildet, die diese Blätter für die Schul/ für die Kita vorbereitet hat und entwickelt hat." (Kita-Leitung $2 \$ 890$ )

Es wurde also etwas aufgegriffen, was in der Schule schon vorhanden war, und für die Bedarfe am Übergang Kita-Schule angepasst. Die Pädagogische Werkstatt begleitete die Erarbeitung der Einlegeblätter. Ebenso wie beim schulischen Portfolio wurden die grafische Gestaltung sowie der Druck mit dem Entwicklungsbudget finanziert. Eine weitere Folge war, dass in einer Arbeitsgruppe, bestehend aus Erzieherinnen aus vier Krippen, das Krippen-Eingewöhnungsbuch erstellt wurde. Damit reicht - bildlich gesprochen - der "rote Faden“ der Beobachtung, Dokumentation und Reflexion von Lernprozessen von der Schule bis in den Krippenbereich.

Ein anderes wichtiges Ergebnis der Evaluation war die Bedeutung des Raums für Kooperation und Innovation. Die gerade skizzierte Entwicklungsarbeit fand zu einem Großteil in den Räumen der Pädagogischen Werkstatt statt, und den Aussagen der Beteiligten zufolge war dies ein wichtiges Element; so betonte eine Lehrerin:

LK-SEK: „Aber auch dieses Schaffen der Räumel also, alleine jetzt zum Beispiel HIER, außerhalb der Schule, diese Werkstatt [...] schafft schon einen angenehmeren Rahmen, und, [...] ja, es ist eine andere Atmosphäre vorhanden, als wenn man sich noch direkt nach der Schule in einem Klassenzimmer oder Lehrerbesprechungszimmer triff, und, ja, gibt einem vielleicht auch ein bisschen Abstand, aus einer anderen Perspektive bestimmte Sachen anzudenken, oder $z u$ (weiter zu besprechen?)“ (Lehrkräfte Sekundarbereich $\$ 300)$.

15 Zur Veranschaulichung: Zu Beginn der Basisdatenerhebung im Schuljahr 2008/2009 befanden sich 41 Kinder in der Schulanfangsphase der Schlüsselgrundschule. Im Schuljahr 2012/13 waren es 62 Kinder (Basisdaten, 2013, unveröff.) und im Schuljahr 2013/2014 70 Kinder (Bericht Transferüberlegungen, Oktober 2013, unveröff.). 
An diesem Ort konnten Befragte aus allen Personengruppen mit Distanz zu ihrem jeweiligen pädagogischen Alltag konzentriert in einer angenehmen Atmosphäre ihre Bildungspraxis reflektieren, diskutieren und gemeinsam mit anderen weiterentwickeln. Hervorgehoben wurde auch die „Neutralität“ des Ortes: Mit der Pädagogischen Werkstatt wurden keine spezifischen Trägerinteressen verbunden, so dass keine Sorge bestand, in die eine oder andere Richtung gelenkt zu werden.

Die hier beschriebenen und in den Interviews kommentierten Prozesse verliefen weder gradlinig noch ohne Hindernisse. Hürden für die Entwicklungsarbeit waren insbesondere die strukturellen Bedingungen, die mangelnde Zeit und das fehlende Personal. Durch die Fusion der drei Schulen und die Bildung der Gemeinschaftsschule z. B. mussten drei Kollegien unterschiedlicher Schulstufen und -formen und mit unterschiedlichen professionellen Selbstverständnissen zusammengebracht werden. Dies gestaltete sich als ein Prozess, der Zeit in Anspruch nahm und weiterhin nimmt, denn er war zum Zeitpunkt der Evaluation noch nicht abgeschlossen und ist es auch aktuell noch nicht. Dies ist nach Ansicht von Befragten auch ein Grund dafür, warum es noch Zeit bedarf, die hier dargestellten Instrumente im gesamten Sekundarbereich der Schule einzuführen.

\section{Fazit}

In diesem Beitrag wurden Ergebnisse der Evaluation von km2 Berlin-Neukölln und zur Pädagogischen Werkstatt vorgestellt und im Zusammenhang mit der Entwicklung von Instrumenten zur Dokumentation und Reflexion der Lernprozesse von Kindern und Jugendlichen im Bildungsnetzwerk km2 Berlin-Neukölln beleuchtet. Es konnte exemplarisch gezeigt werden, welche Funktion die Pädagogische Werkstatt im Bildungsnetzwerk übernommen hat und welche Bedeutung ihr zukommt. Die Evaluationsergebnisse erlauben aufgrund der zur Verfügung stehenden Daten keine statistisch abgesicherten Aussagen darüber, ob und inwiefern das Bildungsnetzwerk km2 Berlin-Neukölln zur Verbesserung der Bildungssituation von Kindern und Jugendlichen im Quartier generell beigetragen hat. Ersichtlich wurde jedoch, dass sich in zehn Jahren km2 Berlin-Neukölln die Bildungspraxis im Hinblick auf die Individualisierung von Bildung aus Sicht der Befragten verändert hat und dass die Pädagogische Werkstatt hierzu einen wesentlichen Beitrag geleistet hat. Es wurden pädagogisch-methodische und personelle Verbindungen innerhalb der Bildungseinrichtungen sowie zwischen den Bildungsstufen und -einrichtungen hergestellt und in dieser Vernetzung eine durchgängige Begleitung der individuellen Bildungsprozesse von Kindern und Jugendlichen erreicht. Mit der Entwicklung und Einführung der aufeinander bezogenen Beobachtungs- und Dokumentationsinstrumente ist in $\mathrm{km} 2$ Berlin-Neukölln etwas gelungen, was in Deutschland 
noch nicht etabliert ist (vgl. Kordulla \& Büker, 2015; Hanke, Backhaus \& Bogatz, 2013).

Im Rückblick wird deutlich, dass für die Befragten die Pädagogische Werkstatt das Synonym für Vernetzung und (interinstitutionelle) kooperative Entwicklungsarbeit darstellt, angefangen von der Identifizierung von Problemen und deren Ursachen bis hin zur Entwicklung der notwendigen Instrumente und Verfahren, um jedes Kind nach seinen Möglichkeiten zu unterstützen. Praxisbegleitung durch ein multiprofessionelles Team, ein von den beteiligten Institutionen unabhängiger Ort und ein eigenes Entwicklungsbudget haben im Zusammenspiel mit den verschiedenen Bildungseinrichtungen und Bildungspartnern die beschriebenen Veränderungen ermöglicht.

Weitere Analysen der vorliegenden Daten, für die etwa theoretische Konzepte zum (regionalen) Netzwerkmanagement und zur Unterstützung im Sinne von Järvinen et al. (2015) herangezogen werden, können zusätzlichen Aufschluss über die Funktion und Bedeutung eines „neutralen, multiprofessionellen Ortes“ wie der Pädagogischen Werkstatt für den Aufbau und die Etablierung von Bildungsnetzwerken bieten, der dazu beitragen (soll), dass „kein Kind und kein Jugendlicher verloren geht“.

\section{Literatur und Internetquellen}

Autorengruppe Bildungsberichterstattung (2016). Bildung in Deutschland 2016. Ein indikatorengestützter Bericht mit einer Analyse zu Bildung und Migration. Bielefeld: wbv.

Baumheier, U., Fortmann, C., \& Warsewa, G. (2013). Ganztagsschulen in lokalen Bildungsnetzwerken. Wiesbaden: Springer VS. https://doi.org/10.1007/978-3-531-19596-4.

Berkemeyer, N., \& Bos, W. (2010). Netzwerke als Gegenstand erziehungswissenschaftlicher Forschung. In C. Stegbauer (Hrsg.), Handbuch Netzwerkforschung (S. 755-770). Wiesbaden: VS. https://doi.org/10.1007/978-3-531-92575-2_66.

Bleckmann, P., \& Durdel, A. (2009). Einführung: Lokale Bildungslandschaften - die zweifache Öffnung. In P. Bleckmann \& A. Durdel (Hrsg.), Lokale Bildungslandschaften. Perspektiven für Ganztagsschulen und Kommunen (S. 11-16). Wiesbaden: VS. https:// doi.org/10.1007/978-3-531-91857-0.

BMFSFJ (Bundesministerium für Familie, Senioren, Frauen und Jugend) (2006). 12. Kinderund Jugendbericht. Berlin: BMFSFJ.

Bollweg, P., \& Otto, H.-U. (Hrsg.). (2011). Räume flexibler Bildung. Bildungslandschaft in der Diskussion. Wiesbaden: VS.

Bronfenbrenner, U., \& Morris, P. A. ( $\left.{ }^{6} 2006\right)$. The Bioecological Model of Human Development. In R. M. Lerner \& W. Damon (Hrsg.), Handbook of Child Psychology (S. 793828). Hoboken, NJ: John Wiley \& Sons Inc.

Deutscher Städtetag (2007). Aachener Erklärung des Deutschen Städtetages anlässlich des Kongresses „Bildung in der Stadt" am 22./23. November 2007. Aachen: Deutscher Städtetag. Zugriff am 12.06.2018. Verfügbar unter: http://www.staedtetag.de/fachinfor mationen/bildung/058050/index.html.

Duveneck, A. (2016). Bildungslandschaften verstehen. Zum Einfluss von Wettbewerbsbedingungen auf die Praxis. Weinheim: Beltz Juventa. 
| Tanja Salem

Hanke, P., Backhaus, J., \& Bogatz, A. (2013). Den Übergang gemeinsam gestalten. Kooperation und Bildungsdokumentation im Übergang von der Kindertageseinrichtung in die Grundschule. Münster: Waxmann.

Järvinen, H., Sendzik, N., Sartory, K., \& Otto, J. (2015). Unterstützungssysteme im Kontext von Regionalisierungsprozessen. Eine theoretische und empirische Annäherung. Journal for Educational Research Online, 7 (1), 94-124.

Jungermann, A., Manitius, V., \& Berkemeyer, N. (2015). Regionalisierung im schulischen Kontext. Ein Überblick zu Projekten und Forschungsbefunden. Journal for Educational Research Online, 7 (1), 14-48.

Kordulla, A., \& Büker, P. (2015). Fachliche und bildungspolitische Ansprüche an eine ressourcenorientierte Bildungsdokumentation. In P. Büker (Hrsg.), Kinderstärken - Kinder stärken. Erziehung und Bildung ressourcenorientiert gestalten (S.135-145). Stuttgart: Kohlhammer.

Kuckartz, U. (22014). Qualitative Inhaltsanalyse. Methoden, Praxis, Computerunterstützung. Weinheim: Beltz Juventa.

Löw, M. (2010). Stadt- und Raumsoziologie. In G. Kneer \& M. Schroer (Hrsg.), Handbuch spezielle Soziologien (S. 605-622). Wiesbaden: VS. https://doi.org/10.1007/978-3-53192027-6_35.

Meinz, M., \& Wenzel, S. (2012). km2 Tools: Basisdatenerhebung. Weinheim: Freudenberg Stiftung.

Million, A., Coelen, T., \& Heinrich, A.J. (2017). Gebaute Bildungslandschaften. Verflechtungen zwischen Pädagogik und Stadtplanung. Berlin: Jovis.

Otto, J., Sendzik, N., Järvinen, H., Berkemeyer, N., \& Bos, W. (2015). Kommunales Netzwerkmanagement. Forschung, Praxis, Perspektiven. Münster: Waxmann.

Rimm-Kaufman, S.E., \& Pianta, R.C. (2000). An Ecological Perspective on the Transition to Kindergarten. Journal of Applied Developmental Psychology, 21 (5), 491-511. https:// doi.org/10.1016/S0193-3973(00)00051-4.

Salem, T. (2018). Ein Quadratkilometer Berlin-Neukölln: Ergebnisse der Abschlussevaluation. Weinheim: Freudenberg Stiftung.

Wenzel, S. (2009). Grundlagenpapier Ein Quadratkilometer Bildung. Unveröffentlichtes Dokument.

Tanja Salem, Dr., geb. 1981, wissenschaftliche Mitarbeiterin in der Freudenberg Stiftung.

E-Mail: tanja.salem@uni-hamburg.de

Anschrift: Universität Hamburg, EW 1, Arbeitsbereich: Diversity in Education Research - DivER, Von-Melle-Park 8, 20146 Hamburg 